\title{
The development of autistic social traits across childhood and adolescence in males and females
}

\author{
William Mandy, ${ }^{1}$ Liz Pellicano, ${ }^{2}$ Beate St Pourcain, ${ }^{3}$ David Skuse, ${ }^{4}$ and Jon Heron ${ }^{5}$ \\ ${ }^{1}$ Research Department of Clinical, Educational and Health Psychology, UCL, London, UK; ${ }^{2}$ Department of \\ Educational Studies, Macquarie University, Sydney, NSW, Australia; ${ }^{3}$ Max Planck Institute for Psycholinguistics, \\ Nijmegen, the Netherlands; ${ }^{4}$ Institute of Child Health, UCL, London; ${ }^{5}$ Centre for Academic Mental Health, Population \\ Health Sciences, Bristol Medical School, University of Bristol, Bristol, UK
}

\begin{abstract}
Background: Autism is a dimensional condition, representing the extreme end of a continuum of social competence that extends throughout the general population. Currently, little is known about how autistic social traits (ASTs), measured across the full spectrum of severity, develop during childhood and adolescence, including whether there are developmental differences between boys and girls. Therefore, we sought to chart the trajectories of ASTs in the general population across childhood and adolescence, with a focus on gender differences. Methods: Participants were 9,744 males $(n=4,784)$ and females $(n=4,960)$ from ALSPAC, a UK birth cohort study. ASTs were assessed when participants were aged 7,10,13 and 16 years, using the parent-report Social Communication Disorders Checklist. Data were modelled using latent growth curve analysis. Results: Developmental trajectories of males and females were nonlinear, showing a decline from 7 to 10 years, followed by an increase between 10 and 16 years. At 7 years, males had higher levels of ASTs than females (mean raw score difference $=0.88,95 \%$ CI $[.72,1.04]$ ), and were more likely (odds ratio $[\mathrm{OR}]=1.99 ; 95 \% \mathrm{CI}, 1.82,2.16$ ) to score in the clinical range on the SCDC. By 16 years this gender difference had disappeared: males and females had, on average, similar levels of ASTs (mean difference $=0.00,95 \% \mathrm{CI}[-0.19,0.19])$ and were equally likely to score in the SCDC's clinical range $(\mathrm{OR}=0.91,95 \%$ CI, $0.73,1.10)$. This was the result of an increase in females' ASTs between 10 and 16 years. Conclusions: There are gender-specific trajectories of autistic social impairment, with females more likely than males to experience an escalation of ASTs during early- and midadolescence. It remains to be discovered whether the observed female adolescent increase in ASTs represents the genuine late onset of social difficulties or earlier, subtle, pre-existing difficulties becoming more obvious. Keywords: Autism; autistic traits; developmental trajectory; sex/gender differences; avon longitudinal study of parents and children.
\end{abstract}

\section{Introduction}

Autism Spectrum Disorder (hereafter 'autism') is characterised by often severe and impairing difficulties with social relatedness and social communication (American Psychiatric Association [APA], 2013). Autism is a dimensional condition, which sits at the extreme of a continuum of social impairment that extends throughout the general population (Constantino, 2009). This is suggested by observations in general population samples of a continuous distribution of autistic social traits (ASTs), with no natural cut-point between autism and subclinical social difficulties (Constantino \& Todd, 2003; Skuse et al., 2009). Furthermore, specific cognitive (e.g. BaronCohen, Wheelwright, Hill, Raste, \& Plumb, 2001) and genetic (e.g. Robinson, Koenen, et al., 2011; Robinson, Munir, et al., 2011 Robinson et al., 2016) aetiological risk factors are associated both with autism and social variability within the nonclinical range.

Autistic social traits are a risk factor for the development of a broad array of emotional, behavioural, social and functional difficulties (Lundström et al., 2011; Mandy, Skuse, Steer, St Pourcain, \&

Conflict of interest statement: No conflicts declared.
Oliver, 2013; Oliver, Barker, Mandy, Skuse, \& Maughan, 2011). This association is linear, across the full range of severity: there is not a threshold below which ASTs confer no risk for co-occurring problems (Skuse et al., 2009). Thus, even AST variability within the normal range is relevant to understanding risk and resilience in development.

Despite their importance within developmental psychopathology, relatively little is known about the developmental trajectory of autistic traits. This is in contrast to other conditions, such as conduct problems, depression and anxiety, the developmental courses of which have been studied for several decades (e.g. Cote, Tremblay, Nagin, Zoccolillo, \& Vitaro, 2002; Kovacs, Feinberg, Crouse-Novak, Paulauskas, \& Finkelstein, 1984; Robins, 1966). Most research on the developmental course of autistic symptoms has focused on participants with a clinical diagnosis of autism who, by definition, represent the extreme high end of the autism trait continuum. This work has found a high degree of stability in autistic symptoms over time among autistic children (Fountain, Winter, \& Bearman, 2012; Gotham, Pickles, \& Lord, 2012; Lord, Luyster, Guthrie, \& Pickles, 2012; Szatmari et al., 2015; Venker, Ray-Subramanian, Bolt, \& Ellis Weismer, 2014). Such research provides valuable clinical and theoretical insights 
into autism, but leaves several questions unanswered. First, studies that only include autistic participants cannot test whether subclinical autistic traits also show stability across development. In particular, such investigations are not suited to testing whether autistic traits ever escalate during development, as any individuals showing such a trajectory would be excluded from studies that preselect participants for having high autistic traits (i.e. autism) at baseline. Second, most work on symptom trajectories of autistic people has focused on infancy and early childhood, and so autistic trait development during late childhood and adolescence is currently poorly understood. Third, selecting children diagnosed with autism, which has a male-tofemale ratio of approximately three-to-one (Loomes, Hull, \& Mandy, 2017), limits the number of female participants, and so reduces power for studying any sex/gender differences in AST developmental trajectory.

The small extant literature on the development of autistic traits across the full continuum of severity only partially addresses these gaps in knowledge. In studies that have calculated correlations between autism traits measured at two time points in nonclinical samples, there is evidence for within-person continuity across late childhood and adolescence. In a mixed-age (8-20 years) sample of male twins $(N=95$ twin pairs), Constantino et al. (2009) observed high autism trait stability $(\mathrm{ICC}=.71)$ over a 5-year period. Similarly, in a larger longitudinal twin study $(N>6,000$ twin pairs), Holmboe et al. (2014) found substantial bivariate correlations for males $(r=.67,95 \%$ CI $[0.66,0.69])$ and females $(r=.60,95 \%$ CI $[0.58,0.62])$ between autism trait scores at 8 and 12 years.

Another study, which examined the association between parent-rated autism traits in toddlerhood and self-reported autism traits in late adolescence, presents a somewhat different picture, having reported a low correlation for males $(r=.16)$ and no association in females $(r=.06$, ns) (Whitehouse, Hickey, \& Ronald, 2011). Yet, the interpretation of these findings is complicated by methodological factors. First, autism traits were measured by parent report at baseline and self-report in adolescence which may have depressed estimates of trait stability. Second, the long time period between the two assessment waves does not allow for a fine-grained understanding of the development of symptoms during this time. For example, the low correlations between toddler and late adolescent autism traits could reflect a general lack of continuity, but would also be compatible with a situation in which there is continuity in childhood, followed by discontinuities in adolescence.

Robinson, Munir, et al. (2011) went some way towards providing a more detailed picture of autism symptom trajectories across childhood and adolescence, as they performed analyses incorporating three time points. They investigated ASTs in a large $(N=6,339)$ general population sample, assessed aged 7,10 and 13 years by parent report. They found within-person stability in ASTs between 7 and 13 years, for both boys and girls. Nevertheless, this belied a shallow, U-shaped AST trajectory, with boys' and girls' scores falling between 7 and 10 years, and rising between 10 and 13 years. It is uncertain whether the rise in ASTs from 10 to 13 years levels off in adolescence, or whether it continues beyond 13 years as an increase in observed ASTs in the teenage years. The current literature reveals little about autism trait trajectories after 13 years. The first aim of the current study is therefore to extend the work of Robinson and colleagues beyond 13 years, to chart the trajectory of ASTs beyond early adolescence.

\section{Sex/Gender differences}

Females who have severe autistic traits are less likely than equivalent males to receive an autism diagnosis (Dworzynski, Ronald, Bolton, \& Happé, 2012; Lai, Lombardo, Auyeung, Chakrabarti, \& Baron-Cohen, 2015; Russell, Steer, \& Golding, 2011). This diagnostic bias is partly due to the existence of a female autism phenotype; a female-specific presentation that is somewhat at odds with the current, malebased, conceptualisation of autism (APA, 2013; Lai et al., 2015). Efforts to reverse current biases against females with high levels of autistic symptoms rely on the development of an empirically based understanding of how autism and autistic traits present in girls and women.

To date, research on sex/gender differences in autistic symptoms has almost exclusively used crosssectional designs (Van Wijngaarden-Cremers et al., 2014). This represents a significant limitation of the literature since a better understanding of sex/gender differences across the life span would help improve recognition and support of autistic difficulties of females. Furthermore, the literature on the developmental course of autism traits across the full range of severity, described above, raises the possibility of gender-specific trajectories: two of the three studies to include both genders reported lower levels of autism trait stability in females than males (Holmboe et al., 2014; Whitehouse et al., 2011). In the current longitudinal study, we critically extend understanding of ASTs in females by investigating gender differences in autism symptom trajectories. We do this by testing the opposing predictions of two hypotheses about the development of the female autistic phenotype.

The female compensation hypothesis states that females with high ASTs learn over time to compensate for their social communication difficulties more effectively than males with high ASTs (Dworzynski et al., 2012; Lai et al., 2015; Mandy et al., 2012). According to this idea, compared to ASTs of males, ASTs in females should diminish during development. 
Support for the female compensation hypothesis comes from the finding that in a sample of autistic adults, retrospective informant reports suggest that females and males experienced similar levels of autistic difficulties in childhood; yet in adulthood, the females demonstrated substantially lower levels of overt autistic social symptoms (Lai et al., 2011). One interpretation of this finding is that severe autistic traits are more likely to diminish over time in females compared to males. A possible mechanism is suggested by the discovery that from childhood onwards, many autistic people learn social coping strategies to mask and compensate for their difficulties (e.g. Hull et al., 2017; Livingston \& Happé, 2017). This is known as social camouflaging, and is more common in females than males (Lai et al., 2017).

An alternative idea is the adolescent emergence hypothesis, which states that compared to males, ASTs in females are more likely to become evident later in development - in adolescence rather than childhood. This idea is based on reports from autistic women that their social and communication difficulties only became overt in adolescence, when their social ecology became increasingly complex (Bargiela, Steward, \& Mandy, 2016); and fits with the observation that autistic females tend to receive their diagnosis later than males (Giarelli et al., 2010). Intriguingly, a version of this as-yet-untested hypothesis was expressed first by Hans Asperger, who wrote "it could be that autistic traits in the female become evident only after puberty. We just do not know" (Asperger, 1944). This hypothesis is in direct contrast to the female compensation hypothesis, which predicts that females' ASTs should diminish from childhood onwards.

In summary, we sought to investigate the course of AST development in the general population between the ages of 7 and 16 years, with a particular focus on determining whether (a) there is an increase in ASTs between 10 and 16 years and (b) AST trajectories between 7 and 16 years differ for males and females.

\section{Methods Procedure}

Participants were enrolled in the Avon Longitudinal Study of Parents and Children (ALSPAC), a general population, longitudinal cohort study in the south-west of England, comprising those with an estimated birth date between April 1991 and December 1992. The total ALSPAC sample comprised 14,541 pregnancies, with 13,971 children alive at 12 months (Boyd et al., 2013). Ethical approval was obtained from the ALSPAC Law and Ethics Committee and Local Research Ethics Committees. Please note that the study website contains details of all the data that are available through a fully searchable data dictionary (http://www.bristol.ac.uk/alspac/researchers).

\section{Participants}

Autistic social traits were measured in ALSPAC at 7, 10, 13 and 16 years. ALSPAC participants were included in the current study if they had AST trait data from at least one of these waves of data collection. Of the 9,744 eligible participants, 95.9\% were white, $2.5 \%$ were born to a teenage mother, $15.2 \%$ had a mother with a university degree and $80.5 \%$ had a parent who was a homeowner. There were no gender differences in any of these demographic characteristics (all ps $>.2$ ). Compared to members of the original birth cohort who are excluded from the current analyses, our participants were more likely to have a mother who: had higher or further education $(\mathrm{OR}=2.36,95 \% \mathrm{CI}$ $[2.15,2.58])$; was a homeowner $(\mathrm{OR}=2.93,95 \% \mathrm{CI}[2.71,3.18])$ and was not a teenager when they gave birth to the participant $(\mathrm{OR}=3.77,95 \% \mathrm{CI}[3.19,4.46])$.

\section{Measures}

Autistic social and communication traits were measured using the Social Communication Disorders Checklist (SCDC), a widely used and well-validated dimensional parent-report measure of ASTs (Skuse, Mandy, \& Scourfield, 2005). The SCDC has excellent reliability (internal consistency $\alpha=.93$, test-retest over 2.7 years $r=.81$ ) (Skuse et al., 2005). Criterion validity is demonstrated by substantial correlations with in-depth, well-validated measures of autistic symptoms (Bölte, Westerwald, Holtmann, Freitag, \& Poustka, 2011; Skuse et al., 2005); and by high sensitivity (.88) and specificity (.91) for autism when a cut-point of 8 or above is used to denote possible caseness (Skuse et al., 2009). Construct validity is shown by the SCDC's high heritability (.74) (Skuse et al., 2005), by its associations with impaired social cognition, pragmatic language impairment, peer problems and antisocial behaviour (convergent validity), and by a lack of/low association with age, socioeconomic status, IQ, social anxiety and teenage pregnancy (discriminant validity) (Bölte et al., 2011; Kothari, Skuse, Wakefield, \& Micali, 2013; Mandy et al., 2013; Pickard, Rijsdijk, Happé, \& Mandy, 2017; Skuse et al., 2005). Further evidence for validity comes from the observation that genetic risk for clinical autism, from common and de novo variants, influences SCDC scores across the full range of severity (Robinson et al., 2016; St Pourcain et al., 2010).

Intelligence was measured aged 8 years using a short form of the Wechsler Intelligence Scales for Children, Third Edition (WISC-III) (Wechsler, 1992), administered by trained psychologists. We categorised participants into low (IQ $<85$ ), average $(85 \leq \mathrm{IQ} \geq 115)$ and high (IQ $>115)$ groups, enabling us to investigate and depict AST trajectories at different levels of IQ. These cut-points were chosen to map onto those used by Robinson, Munir, et al. (2011), to ensure comparability of our work with that study.

\section{Analysis}

Initially, means for the SCDC administered at 7, 10, 13 and 16 years were plotted. These are shown for males and females in Figure 1A. The data were then modelled using various nonlinear latent growth curve models, with latent growth factors (random effects) capturing heterogeneity in both baseline levels and longitudinal patterns of change. Gender differences in patterns of change were studied using a multigroup model, which allowed growth factor means, (co)variances and residual variances to vary between males and females. Time was specified as the number of years since age 7 (with loadings $0.157,3.221,6.406$ and 9.330) and assumed fixed for all individuals. Residual variances were constrained to be longitudinally invariant within each group, the default in multilevel modelling. Subsequently, IQ was included as a covariate to examine the association between IQ and the growth factors for SCDC, again within each gender as this relationship may be different for boys and girls (Skuse et al., 2009).

To retrieve the maximum amount of information possible from the data, a Maximum Likelihood-based approach to 
missing data was adopted permitting partial responders to be included in the sample $(N=9,744)$. These methods make the Missing at Random assumption, namely that there are no systematic differences between observed and missing values for any dependent variable when conditioning on the remaining variables in the model. To investigate the robustness of our models to consequences of missing data, we reran our analyses for those with complete SCDC data $(n=4,380)$, and those with one $(n=6,385)$ or two $(n=8,024)$ of the four SCDC data points missing (see Table S1)

\section{Results}

Figure 1A shows the mean scores for the SCDC at 7, 10, 13 and 16 years. Focussing on the meanstructure residuals from the latent growth models, two alternative formulations appeared suited to capturing the population average change, namely a two-part piecewise linear ('spline') model (with a knot at the second time point) and a quadratic polynomial model. While the two models are of equal parsimony, the piecewise model encountered some estimation problems due to a very strong correlation between the first and second slope growth factors. Consequently, we opted for the model that allowed mean SCDC to change as a quadratic function of age.

We used a multigroup approach and explored a range of models in which the intercept, slope and quadratic term variances and covariances were permitted to vary between genders rather than simply
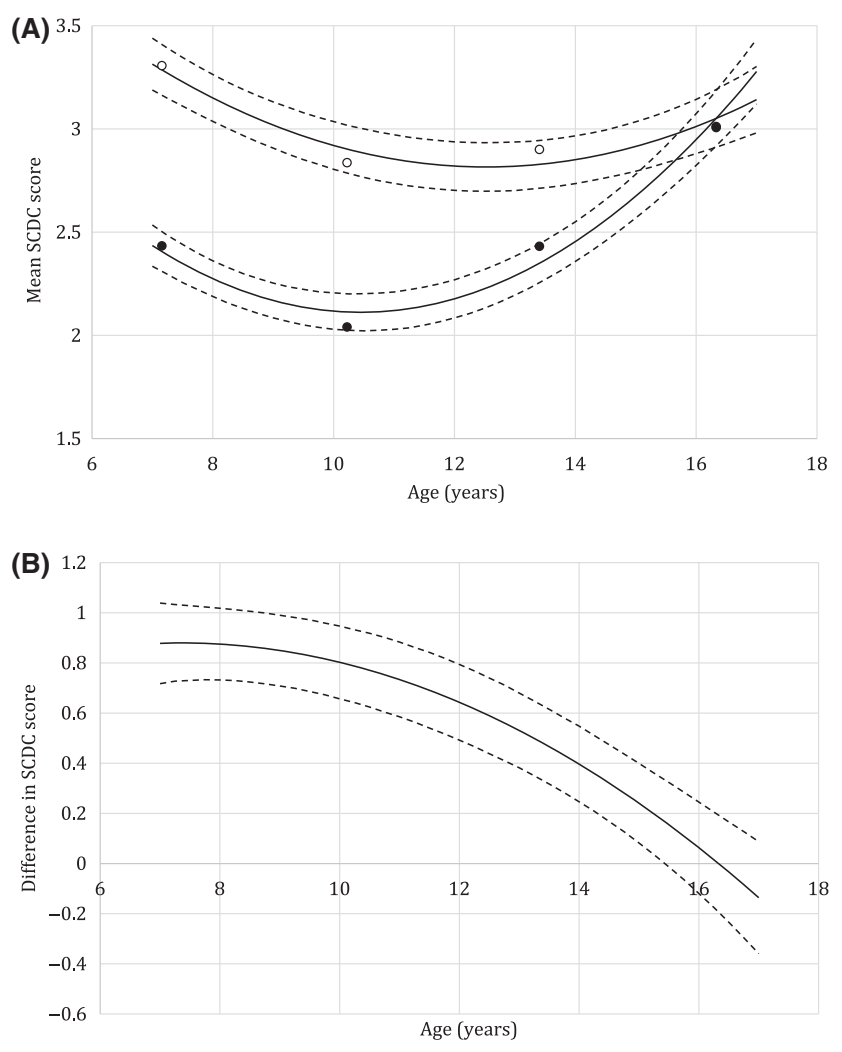

Figure 1 (A) Social Communication Disorders Checklist (SCDC) trajectories for boys (upper curve/white circles) and girls (lower curve/black circles). Note: Circles denote actual sample means. (B) Social Communication Disorders Checklist (SCDC) gender difference across childhood and adolescence the growth factor means. We also considered the possibility that the variance of the occasion-specific (error) residuals may be different for males and females. By studying summary fit statistics (CFI, TLI, RMSEA), comparative fit statistics (sample size adjusted BIC) and the covariance structure residuals we opted for the model in which all variance and covariance terms were allowed to be gender variant (see Tables S1 and S2).

Predicted scores from this model are presented in Table 1, and depicted in Figure 1A, demonstrating that from the age of 10 years, there was a narrowing of the gender gap in SCDC scores, due to a sharper increase in average SCDC scores for females compared to males. This is confirmed by Figure 1B, showing the estimated gender difference as a function of age. Here, the tendency for boys to have higher SCDC-measured traits at age seven diminishes over time: by the age of 16 years this gender difference is no longer present.

When we added IQ to the model we did not find evidence that IQ moderated the effect of gender on SCDC score, as tests for an interaction between gender and IQ were not significant at 7, 10, 13 or 16 years (all $p$ values $>$.320) (see Figure $\mathrm{S} 1$ and Table S3).

We were interested to discover whether the pattern of findings for means of continuously measured ASTs held for those with severe difficulties of clinical relevance. To this end we examined the proportion of males and females scoring above the conventional clinical cut-point of the SCDC (i.e. 8) at 7, 10, 13 and 16 years. We also inspected alternative, more stringent cut-points, namely the 95th and 99th percentile. The results of these analyses are presented in Table 1, and show a similar pattern to the findings of the latent growth curve model: at 7 years, males were substantially more likely than females to score high on the SCDC, but this gender gap declined over time such that by 16 years, males and females were equally likely to show high levels of ASTs, regardless of the threshold used.

In total 1,442 participants scored above the clinical threshold of the SCDC at least once across the four waves of data collection in the current study. The age at which these individuals first scored above threshold is shown in Table 2. Females had higher odds than males of first being identified by the SCDC as having high ASTs in adolescence (at 13 or 16 years) rather than in childhood (at 7 or 10 years) $(\mathrm{OR}=2.47,95 \%$ CI $[1.97,3.09])$.

\section{Discussion}

This was the first investigation of autistic social trait (AST) trajectories in the general population spanning late childhood (7 years) and midadolescence (16 years). We discovered distinct AST trajectories for males and females. At 7 years of age, males had higher ASTs than females; and these showed a 
Table 1 Social Communication Disorders Checklist (SCDC) scores for males and females across childhood and adolescence



SCDC, Social Communication Disorders Checklist; OR, Odds ratio.

Table 2 Age at which first scored above Social Communication Disorders Checklist clinical threshold

\begin{tabular}{lcc}
\hline & $\begin{array}{c}\text { Females } \\
n(\%)\end{array}$ & $\begin{array}{c}\text { Males } \\
n(\%)\end{array}$ \\
\hline 7 years & $209(33.6)$ & $416(51.2)$ \\
10 years & $110(17.7)$ & $172(21.0)$ \\
13 years & $128(20.6)$ & $131(16.0)$ \\
16 years & $176(28.3)$ & $97(11.8)$ \\
Total & 623 & 819 \\
\hline
\end{tabular}

At each time point, individuals with missing data were excluded.

shallow u-shaped curve, declining between 7 and 10 years and then rising slightly from 10 to 16 years. At 16 years, males had, on average, lower ASTs than they had at 7 years. In contrast, females showed a marked escalation in ASTs during adolescence, such that by 16 years females had, on average, higher ASTs than they had at 7 years. By midadolescence, there was no longer a tendency for females to have lower ASTs than males. These findings apply across the IQ range. When we looked at the individuals with very high ASTs the same pattern was observed: in adolescence females caught up with males, such that by 16 years, males and females were at equal likelihood of having clinically severe levels of ASTs.

This new finding of an escalation of parentobserved ASTs in females during adolescence contradicts the notion that females' ASTs decline over time as they learn strategies to cope with and mask their social difficulties ('female compensation hypothesis'). Instead, the trajectories we observed are consistent with the 'adolescent emergence hypothesis', first proposed by Hans Asperger but never previously tested, that female ASTs appear later than those of males (Asperger, 1944). It is interesting to note that the female teenage increase in ASTs that we report for the first time has been observed for another aspect of atypical neurodevelopment: over the course of adolescence the male-tofemale ratio for attention deficit/hyperactivity disorder (ADHD) narrows, reflecting an increase in observable symptoms in females during this period (Zahn-Waxler, Crick, Shirtcliff, \& Woods, 2006).

One interpretation of the female increase in AST scores in adolescence is that there are some females who have subtle social difficulties that went unnoticed by their parents in childhood, but which became problematic, and therefore overt, once they were required to navigate the increasingly complex adolescent social world. This is consistent with the reports of late-diagnosed autistic women, who tend to recall childhood social impairments that only became severely impairing during adolescence (Bargiela et al., 2016). Of relevance to our observation of AST increases in females but not males, there is some evidence that the rise in social demands upon 
adolescents is especially pronounced for females (Dunn, 2004).

Another interpretation of the increase in observed ASTs of females in adolescence is that it reflects the genuine late onset of new problems that were not present in childhood. One possibility for future investigation is that the biological changes of puberty could be implicated. A first step will be testing whether the increase in parent-reported ASTs in females is specifically associated with progression through puberty, rather than chronological age. If this is the case, it will be valuable to consider how ovarian hormones like oestrogen could influence ASTs, for example, via its downstream effects on serotonin and oxytocin, and on the hypothalimicpituitary axis (Martel, 2013). A related possibility is that a 'two-hit' mechanism could be in operation, whereby pubertal influences interact with pre-existing vulnerabilities (Picci \& Scherf, 2015). Furthermore, there is evidence that some genetic influences on social behaviour only start to influence the phenotype in adolescence, and it will be important to investigate whether such time-specific genetic effects are moderated by gender (St Pourcain, Eaves, et al., 2017; St Pourcain, Robinson et al., 2017).

Another key question is whether the SCDC-measured traits we observed in adolescent girls are truly autistic in nature, or whether they represent a different type of social impairment, for example, arising from the onset of internalising problems, ADHD or psychosis risk (St Pourcain, Eaves, et al., 2017; St Pourcain, Robinson et al., 2017). Several strands of evidence support the notion that the female social variability we observed in adolescence is related to autism. First, our findings are compatible with observations from cross-sectional studies using alternative AST measures, which find females have lower levels of ASTs than males in child samples (Constantino \& Todd, 2003), but that this gender difference is attenuated, or nonexistent in adult samples (e.g. Kanne, Christ, \& Reiersen, 2009; Takei et al., 2014). Second, the SCDC is a wellvalidated measure that continues to show high heritability in adolescence, as would be expected of a true measure of ASTs (St Pourcain et al., 2014). Third, the SCDC has been shown to measure social communication difficulties independent of other types of psychopathology throughout childhood and adolescence (Mandy et al., 2013; Oliver et al., 2011; Robinson, Koenen, et al., 2011; Robinson, Munir, et al., 2011; Skuse et al., 2009). In particular, using a cross-lagged model, Pickard et al. (2017) showed that in adolescence SCDC scores predict subsequent increases in anxiety symptoms; but that the reverse is not true. This contradicts the idea that the rise in SCDC scores of females we observed is merely an epiphenomenon driven by a rise in internalising problems (in this case, social anxiety). Nevertheless, future work is needed to elucidate the nature and causes of adolescent onset social communication difficulties, including the extent to which they are influenced by nonautistic factors (St Pourcain, Eaves, et al., 2017; St Pourcain, Robinson et al., 2017). This should include investigation of their infant and early childhood precursors, co-occurring psychopathology and early adult outcomes.

\section{Limitations}

The findings of this study should be considered in the light of the following limitations. First, our measure of ASTs is focused on the core social difficulties of autism, not those concerning inflexibility and atypical sensory processing. Therefore, it remains unclear whether the pattern of findings we report would also be observed for these 'non-social' elements of autism. Longitudinal investigations using trait measures such as the Social Responsiveness Scale (Constantino \& Todd, 2003), which span childhood and adolescence and measure the full array of autistic features, are needed. Second, like all large birth cohort studies, ALSPAC has suffered attrition, with families of higher socioeconomic status (SES) more likely to participate, which could affect generalisability of findings. Nevertheless, our findings were robust to missing data. Third, our analyses only extend to midadolescence. It will be important in future to chart AST trajectories for males and females during the transition from adolescence to adulthood. Fourth, like most studies of AST in the general population of young people, our measure relied upon parent report. While parent report is more sensitive than teacher report (Posserud, Lundervold, \& Gillberg, 2006) and direct observation (Lai et al., 2011) to autistic traits in females, it will nevertheless be valuable in future to triangulate our findings using different methods of assessment, especially self-report. Such work can help elucidate whether our finding of an increase in female ASTs could partially reflect changes in parental expectations. For example, it may be that parental expectations of their daughters' social skills increase more substantially than of their sons' during adolescence.

\section{Conclusions}

Our findings have the following clinical implications. First, they likely help explain the fact that autistic females receive their diagnosis later on average than autistic males, and are more likely to reach adulthood without appropriate understanding and support (Bargiela et al., 2016; Giarelli et al., 2010). They also fit with the observation that the male-to-female ratio is lower in adult and adolescent autism clinics ( 2-to-1) compared to child ones ( 6-to-1) (Rutherford et al., 2016). It is important that educational and clinical services are responsive to the fact that some autistic females may first present in 
adolescence, after the age at which most autism cases are identified, without an extensive history of obvious childhood autistic difficulties. Second, the underdiagnosis of females with high ASTs, relative to high AST males, has sometimes been attributed to their having a greater capacity to compensate for their core autistic difficulties (e.g. Dworzynski et al., 2012; Mandy et al., 2012). Our findings contradict this idea, as they show that ASTs in females tend to get worse, not better, over time. This suggests the need to ensure that high-AST females receive appropriate clinical diagnoses and associated support.

The analyses presented emphasise the value of studying the 'chronogeneity' (i.e. longitudinal heterogeneity) of autistic symptoms (Georgiades, Bishop, \& Frazier, 2017). A next step will be to use growth mixture modelling to identify specific subgroups defined by AST trajectory, and to identify the clinical utility of these groups. This would extend our understanding of which children, and in particular which girls, are likely to require clinical and/or educational support due to having social communication difficulties.

\section{Supporting information}

Additional Supporting Information may be found in the online version of this article:

Table S1. Fit statistics for latent growth curve models under different missing data conditions.
Table S2. Parameters of chosen model (fully parameter variant/varying Q) for boys and girls under different missing data conditions.

Table S3. Model-estimated means by IQ and gender (all available data on SCDC and IQ, $n=6,872$ ).

Figure S1. Social Communication Disorders Checklist trajectories for boys and girls by IQ.

\section{Acknowledgements}

The UK Medical Research Council and Wellcome (Grant ref: 102215/2/13/2) and the University of Bristol provide core support for ALSPAC. The authors are extremely grateful to all the families who took part in this study, the midwives for their help in recruiting them and the whole ALSPAC team, which includes interviewers, computer and laboratory technicians, clerical workers, research scientists, volunteers, managers, receptionists and nurses. They also thank Dr Meng-Chuan Lai (University of Toronto) and Dr Stelios Georgiades (McMaster University) for their valuable insights during discussion of the work presented in this manuscript. The authors have declared that they have no competing or potential conflicts of interest.

\section{Correspondence}

William Mandy, UCL Research Department of Clinical, Educational and Health Psychology, Gower Street, London, WC1N 6BT, UK; Email: w.mandy@ucl.ac.uk

\section{Key points}

- Autism is a dimensional condition, representing the extreme of a continuum of social competence that extends throughout the population: where an individual sits on this autistic social trait (AST) continuum predicts their chances of adaptation and well-being.

- Despite their importance in developmental psychopathology, little is known about the developmental course of ASTs in the general population of children and adolescents.

- Autistic social trait trajectories were different for males and females. Aged 7 years, boys had, on average higher levels of ASTs than girls. By midadolescence, this gender difference had disappeared, due to a marked escalation of ASTs of females between 10 and 16 years.

- Of those with clinically severe ASTs, $37 \%$ did not show these before 13 years. The majority (57\%) of this adolescent onset group were female.

- Clinical services and educational systems should be responsive to social communication difficulties that only become overt in adolescence, especially in females.

\section{References}

American Psychiatric Association (2013). Diagnostic and statistical manual (DSM-5) (5th edn). Arlington, VA: Author.

Asperger, H. (1944). Die 'Autistischen psychopathen' im kindesalter. Archiv für Psychiatrie und Nervenkrankheiten, 117, 76-136.

Bargiela, S., Steward, R., \& Mandy, W. (2016). The experiences of late-diagnosed women with autism spectrum conditions: An investigation of the female autism phenotype. Journal of Autism and Developmental Disorders, 46, 3281-3294.
Baron-Cohen, S., Wheelwright, S., Hill, J., Raste, Y., \& Plumb, I. (2001). The 'Reading the Mind in the Eyes' test revised version: A study with normal adults, and adults with Asperger syndrome or high-functioning autism. Journal of Child Psychology and Psychiatry, 42, 241-251.

Bölte, S., Westerwald, E., Holtmann, M., Freitag, C., \& Poustka, F. (2011). Autistic traits and autism spectrum disorders: The clinical validity of two measures presuming a continuum of social communication skills. Journal of Autism and Developmental Disorders, 41, 66-72.

Boyd, A., Golding, J., Macleod, J., Lawlor, D.A., Fraser, A., Henderson, J., . . \& Davey Smith, G. (2013). Cohort Profile: 
The 'children of the 90s'-the index offspring of the Avon Longitudinal Study of Parents and Children. International Journal of Epidemiology, 42, 111-127.

Constantino, J.N. (2009). How continua converge in nature: Cognition, social competence, and autistic syndromes. Journal of the American Academy of Child and Adolescent Psychiatry, 48, 97-98.

Constantino, J.N., Abbacchi, A.M., Lavesser, P.D., Reed, H., Givens, L., Chiang, L., ... \& Todd, R.D. (2009). Developmental course of autistic social impairment in males. Development and Psychopathology, 21, 127-138.

Constantino, J.N., \& Todd, R.D. (2003). Autistic traits in the general population: A twin study. Archives of General Psychiatry, 60, 524-530.

Cote, S., Tremblay, R.E., Nagin, D., Zoccolillo, M., \& Vitaro, F. (2002). The development of impulsivity, fearfulness, and helpfulness during childhood: Patterns of consistency and change in the trajectories of boys and girls. Journal of Child Psychology and Psychiatry, 43, 609-618.

Dunn, J. (2004). Children's friendships: The beginnings of intimacy. Malden, MA: Blackwell.

Dworzynski, K., Ronald, A., Bolton, P., \& Happé, F. (2012). How different are girls and boys above and below the diagnostic threshold for autism spectrum disorders? Journal of the American Academy of Child and Adolescent Psychiatry, 51, 788-797.

Fountain, C., Winter, A.S., \& Bearman, P.S. (2012). Six developmental trajectories characterize children with autism. Pediatrics, 129, e1112-e1120.

Georgiades, S., Bishop, S.L., \& Frazier, T. (2017). Editorial Perspective: Longitudinal research in autism - introducing the concept of 'chronogeneity'. Journal of Child Psychology and Psychiatry, and Allied Disciplines, 58, 634-636.

Giarelli, E., Wiggins, L.D., Rice, C.E., Levy, S.E., Kirby, R.S., Pinto-Martin, J., \& Mandell, D. (2010). Sex differences in the evaluation and diagnosis of autism spectrum disorders among children. Disability and Health Journal, 3, 107-116.

Gotham, K., Pickles, A., \& Lord, C. (2012). Trajectories of autism severity in children using standardized ADOS scores. Pediatrics, 130, e1278-e1284.

Holmboe, K., Rijsdijk, F.V., Hallett, V., Happé, F., Plomin, R., \& Ronald, A. (2014). Strong genetic influences on the stability of autistic traits in childhood. Journal of the American Academy of Child \& Adolescent Psychiatry, 53, 221-230.

Hull, L., Petrides, K.V., Allison, C., Smith, P., Baron-Cohen, S., Lai, M.C., \& Mandy, W. (2017). "Putting on my best normal": Social camouflaging in adults with autism spectrum conditions. Journal of Autism and Developmental Disorders, 47, 2519-2534.

Kanne, S.M., Christ, S.E., \& Reiersen, A.M. (2009). Psychiatric symptoms and psychosocial difficulties in young adults with autistic traits. Journal of Autism and Developmental Disorders, 39, 827-833.

Kothari, R., Skuse, D., Wakefield, J., \& Micali, N. (2013). Gender differences in the relationship between social communication and emotion recognition. Journal of the American Academy of Child and Adolescent Psychiatry, 52, 11481157.e2.

Kovacs, M., Feinberg, T.L., Crouse-Novak, M.A., Paulauskas, S.L., \& Finkelstein, R. (1984). Depressive disorders in childhood: I. A longitudinal prospective study of characteristics and recovery. Archives of General Psychiatry, 41, 229237.

Lai, M.-C., Lombardo, M.V., Auyeung, B., Chakrabarti, B., \& Baron-Cohen, S. (2015). Sex/gender differences and autism: Setting the scene for future research. Journal of the American Academy of Child and Adolescent Psychiatry, 54, 11-24.

Lai, M.C., Lombardo, M.V., Pasco, G., Ruigrok, A.N., Wheelwright, S.J., Sadek, S.A., ... \& MRC AIMS Consortium (2011). A behavioral comparison of male and female adults with high functioning autism spectrum conditions. PLoS ONE, 6, e20835.

Lai, M.C., Lombardo, M.V., Ruigrok, A.N., Chakrabarti, B., Auyeung, B., Szatmari, P., ... \& MRC AIMS Consortium (2017). Quantifying and exploring camouflaging in men and women with autism. Autism, 21, 690-702.

Livingston, L.A., \& Happé, F. (2017). Conceptualising Compensation in Neurodevelopmental. American Journal of Medical Genetics. Part C, Seminars in Medical Genetics, 169, 198-208.

Loomes, R., Hull, L., \& Mandy, W.P.L. (2017). What is the male-to-female ratio in autism spectrum disorder? A systematic review and meta-analysis. Journal of the American Academy of Child and Adolescent Psychiatry, 56, 466-474.

Lord, C., Luyster, R., Guthrie, W., \& Pickles, A. (2012). Patterns of developmental trajectories in toddlers with autism spectrum disorder. Journal of Consulting and Clinical Psychology, 80, 477-489.

Lundström, S., Chang, Z., Kerekes, N., Gumpert, C.H., Råstam, M., Gillberg, C., ... \& Anckarsäter, H. (2011). Autistic-like traits and their association with mental health problems in two nationwide twin cohorts of children and adults. Psychological Medicine, 41, 2423-2433.

Mandy, W., Chilvers, R., Chowdhury, U., Salter, G., Seigal, A., \& Skuse, D. (2012). Sex differences in autism spectrum disorder: Evidence from a large sample of children and adolescents. Journal of Autism and Developmental Disorders, 42, 1304-1313.

Mandy, W., Skuse, D., Steer, C., St Pourcain, B., \& Oliver, B.R. (2013). Oppositionality and socioemotional competence: Interacting risk factors in the development of childhood conduct disorder symptoms. Journal of the American Academy of Child and Adolescent Psychiatry, 52, 718-727.

Martel, M.M. (2013). Sexual selection and sex differences in the prevalence of childhood externalizing and adolescent internalizing disorders. Psychological Bulletin, 139, 1221.

Oliver, B.R., Barker, E.D., Mandy, W.P.L., Skuse, D.H., \& Maughan, B. (2011). Social cognition and conduct problems: A developmental approach. Journal of the American Academy of Child and Adolescent Psychiatry, 50, 385-394.

Picci, G., \& Scherf, K.S. (2015). A two-hit model of autism: Adolescence as the second hit. Clinical Psychological Science, 3, 349-371.

Pickard, H., Rijsdijk, F., Happé, F., \& Mandy, W. (2017). Are social and communication difficulties a risk factor for the development of social anxiety? Journal of the American Academy of Child \& Adolescent Psychiatry, 56, 344-351.e3.

Posserud, M.B., Lundervold, A.J., \& Gillberg, C. (2006). Autistic features in a total population of 7-9-year-old children assessed by the ASSQ (Autism Spectrum Screening Questionnaire). Journal of Child Psychology and Psychiatry, 47, 167-175.

Robins, L. (1966). Deviant children grow up: A sociological and psychiatric study of sociopathic personality. Baltimore: Williams \& Wilkins.

Robinson, E.B., Koenen, K.C., McCormick, M.C., Munir, K., Hallett, V., Happé, F., .. \& \&onald, A. (2011). Evidence that autistic traits show the same etiology in the general population and at the quantitative extremes $(5 \%, 2.5 \%$, and $1 \%)$. Archives of General Psychiatry, 68, 1113-1121.

Robinson, E.B., Munir, K., Munafò, M.R., Hughes, M., McCormick, M.C., \& Koenen, K.C. (2011). Stability of autistic traits in the general population: Further evidence for a continuum of impairment. Journal of the American Academy of Child \& Adolescent Psychiatry, 50, 376-384.

Robinson, E.B., St Pourcain, B., Anttila, V., Kosmicki, J.A., Bulik-Sullivan, B., Grove, J., . . \& \& Daly, M.J. (2016). Genetic risk for autism spectrum disorders and neuropsychiatric variation in the general population. Nature Genetics, 48, $552-555$ 
Russell, G., Steer, C., \& Golding, J. (2011). Social and demographic factors that influence the diagnosis of autistic spectrum disorders. Social Psychiatry and Psychiatric Epidemiology, 46, 1283-1293.

Rutherford, M., McKenzie, K., Johnson, T., Catchpole, C., O'Hare, A., McClure, I., \& Murray, A. (2016). Gender ratio in a clinical population sample, age of diagnosis and duration of assessment in children and adults with autism spectrum disorder. Autism, 20, 628-634.

Skuse, D.H., Mandy, W.P.L., \& Scourfield, J. (2005). Measuring autistic traits: Heritability, reliability and validity of the Social and Communication Disorders Checklist. The British Journal of Psychiatry, 187, 568-572.

Skuse, D.H., Mandy, W., Steer, C., Miller, L.L., Goodman, R., Lawrence, K., . . \& Golding, J. (2009). Social communication competence and functional adaptation in a general population of children: Preliminary evidence for sex-by-verbal IQ differential risk. Journal of the American Academy of Child and Adolescent Psychiatry, 48, 128-137.

St Pourcain, B., Eaves, L.J., Ring, S.M., Fisher, S.E., Medland, S., Evans, D.M., \& Smith, G.D. (2017). Developmental changes within the genetic architecture of social communication behaviour: A multivariate study of genetic variance in unrelated individuals. Biological Psychiatry, 83, 598-606.

St Pourcain, B., Robinson, E.B., Anttila, V., Sullivan, B.B., Maller, J., Golding, J., .. \& \& Davey Smith, G. (2017). ASD and schizophrenia show distinct developmental profiles in common genetic overlap with population-based social communication difficulties. Molecular Psychiatry, 23, 263-270.

St Pourcain, B., Skuse, D.H., Mandy, W.P., Wang, K., Hakonarson, H., Timpson, N.J., .. \& Smith, G.D. (2014). Variability in the common genetic architecture of socialcommunication spectrum phenotypes during childhood and adolescence. Molecular Autism, 5, 18.

St Pourcain, B., Wang, K., Glessner, J.T., Golding, J., Steer, C., Ring, S.M., ... \& Davey Smith, G. (2010). Association between a high-risk autism locus on 5 p14 and social communication spectrum phenotypes in the general population. The American Journal of Psychiatry, 167, 1364-1372.

Szatmari, P., Georgiades, S., Duku, E., Bennett, T.A., Bryson, S., Fombonne, E., . . \& Pathways in ASD Study Team (2015). Developmental trajectories of symptom severity and adaptive functioning in an inception cohort of preschool children with autism spectrum disorder. JAMA Psychiatry, 72, 276283.

Takei, R., Matsuo, J., Takahashi, H., Uchiyama, T., Kunugi, H., \& Kamio, Y. (2014). Verification of the utility of the social responsiveness scale for adults in non-clinical and clinical adult populations in Japan. BMC Psychiatry, 14, 302.

Venker, C.E., Ray-Subramanian, C.E., Bolt, D.M., \& Ellis Weismer, S. (2014). Trajectories of autism severity in early childhood. Journal of Autism and Developmental Disorders, 44, 546-563.

Wechsler, D. (1992). Manual for the wechsler intelligence scale for children-third UK Edition (WISC-III UK). Kent, UK: Psychological Corporation.

Whitehouse, A.J., Hickey, M., \& Ronald, A. (2011). Are autistic traits in the general population stable across development? PLoS ONE, 6, e23029.

van Wijngaarden-Cremers, P.J.M., van Eeten, E., Groen, W.B., van Deurzen, P.A., Oosterling, I.J., \& Van der Gaag, R.J. (2014). Gender and age differences in the core triad of impairments in autism spectrum disorders: A systematic review and meta-analysis. Journal of Autism and Developmental Disorders, 44, 627-635.

Zahn-Waxler, C., Crick, N.R., Shirtcliff, E.A., \& Woods, K.E. (2006). The origins and development of psychopathology in females and males. In D. Cicchetti \& D.J. Cohen (Eds.), Developmental psychopathology: Theory and method (pp. 76-138). Hoboken, NJ: John Wiley.

Accepted for publication: 28 February 2018

First published online: 19 April 2018 